\title{
On Fibonacci functions with periodicity
}

\author{
Hee Sik Kim ${ }^{1}$, Joseph Neggers ${ }^{2}$ and Keum Sook So ${ }^{3 *}$
}

"Correspondence:

ksso@hallym.ac.kr

${ }^{3}$ Department of Mathematics, Hallym University, Chuncheon,

200-702, Korea

Full list of author information is

available at the end of the article

\begin{abstract}
In this paper we discuss Fibonacci functions using the (ultimately) periodicity and we also discuss the exponential Fibonacci functions. Especially, given a non-negative real-valued function, we obtain several exponential Fibonacci functions.
\end{abstract}

MSC: $11 \mathrm{~B} 39 ; 39 \mathrm{~A} 10$

Keywords: Fibonacci function; (ultimately) periodic

\section{Introduction}

Fibonacci numbers have been studied in many different forms for centuries and the literature on the subject is consequently incredibly vast. One of the amazing qualities of these numbers is the variety of mathematical models where they play some sort of role and where their properties are of importance in elucidating the ability of the model under discussion to explain whatever implications are inherent in it. The fact that the ratio of successive Fibonacci numbers approaches the golden ratio (section) rather quickly as they go to infinity probably has a good deal to do with the observation made in the previous sentence. Surveys and connections of the type just mentioned are provided in [1] and [2] for a very minimal set of examples of such texts, while in [3] Kim and Neggers showed that there is a mapping $D: M \rightarrow D M$ on means such that if $M$ is a Fibonacci mean so is $D M$, and that if $M$ is the harmonic mean, then $D M$ is the arithmetic mean, and if $M$ is a Fibonacci mean, then $\lim _{n \rightarrow \infty} D^{n} M$ is the golden section mean. The Hyers-Ulam stability of Fibonacci functional equation was studied in [4]. Surprisingly novel perspectives are still available and will presumably continue to be so for the future as long as mathematical investigations continue to be made. In the following the authors of the present paper are making another small offering at the same spot as many previous contributors have visited in both recent and more distant pasts.

Han et al. [5] considered several properties of Fibonacci sequences in arbitrary groupoids. They discussed Fibonacci sequences in both several groupoids and groups. The present authors [6] introduced the notion of generalized Fibonacci sequences over a groupoid and discussed these in particular for the case where the groupoid contains idempotents and pre-idempotents. Using the notion of Smarandache-type $P$-algebras they obtained several relations on groupoids which are derived from generalized Fibonacci sequences.

In [7] Han et al. discussed Fibonacci functions on the real numbers $\mathbf{R}$, i.e., functions $f: \mathbf{R} \rightarrow \mathbf{R}$ such that, for all $x \in \mathbf{R}, f(x+2)=f(x+1)+f(x)$, and developed the notion of Fibonacci functions using the concept of $f$-even and $f$-odd functions. Moreover, they showed that if $f$ is a Fibonacci function, then $\lim _{x \rightarrow \infty} \frac{f(x+1)}{f(x)}=\frac{1+\sqrt{5}}{2}$.

(อ2014 Kim et al.; licensee Springer. This is an Open Access article distributed under the terms of the Creative Commons Attribution License (http://creativecommons.org/licenses/by/2.0), which permits unrestricted use, distribution, and reproduction in any medium, provided the original work is properly cited. 
In this paper we discuss Fibonacci functions using the (ultimately) periodicity and we also discuss the exponential Fibonacci functions. Especially, given a non-negative realvalued function, we obtain several exponential Fibonacci functions.

\section{Preliminaries}

A function $f$ defined on the real numbers is said to be a Fibonacci function [7] if it satisfies the formula

$$
f(x+2)=f(x+1)+f(x)
$$

for any $x \in \mathbf{R}$, where $\mathbf{R}$ (as usual) is the set of real numbers.

Example 2.1 ([7]) Let $f(x):=a^{x}$ be a Fibonacci function on $\mathbf{R}$ where $a>0$. Then $a^{x} a^{2}=$ $f(x+2)=f(x+1)+f(x)=a^{x}(a+1)$. Since $a>0$, we have $a^{2}=a+1$ and $a=\frac{1+\sqrt{5}}{2}$. Hence $f(x)=\left(\frac{1+\sqrt{5}}{2}\right)^{x}$ is a Fibonacci function, and the unique Fibonacci function of this type on $\mathbf{R}$.

If we let $u_{0}=0, u_{1}=1$, then we consider the full Fibonacci sequence: ..., 5, -3, 2, -1, 1, 0, 1, $1,2,3,5, \ldots$, i.e., $u_{-n}=(-1)^{n} u_{n}$ for $n>0$, and $u_{n}=F_{n}$, the $n$th Fibonacci number.

Example 2.2([7]) Let $\left\{u_{n}\right\}_{n=-\infty}^{\infty}$ and $\left\{v_{n}\right\}_{n=-\infty}^{\infty}$ be full Fibonacci sequences. We define a function $f(x)$ by $f(x):=u_{\lfloor x\rfloor}+v_{\lfloor x\rfloor} t$, where $t=x-\lfloor x\rfloor \in[0,1)$. Then $f(x+2)=u_{\lfloor x+2\rfloor}+$ $v_{\lfloor x+2\rfloor} t=u_{(\lfloor x\rfloor+2)}+v_{(\lfloor x\rfloor+2)} t=\left(u_{(\lfloor x\rfloor+1)}+u_{\lfloor x\rfloor}\right)+\left(v_{(\lfloor x\rfloor+1)}+v_{\lfloor x\rfloor}\right) t=f(x+1)+f(x)$ for any $x \in \mathbf{R}$. This proves that $f$ is a Fibonacci function.

Example 2.3 Let $\varphi(t), \psi(t)$ be any real-valued-functions defined on $[0,1)$ and let $\left\{u_{n}\right\}_{n=-\infty}^{\infty}$ and $\left\{v_{n}\right\}_{n=-\infty}^{\infty}$ be full Fibonacci sequences, respectively. Define a map $f(x):=u_{\lfloor x\rfloor} \varphi(t)+$ $v_{\lfloor x\rfloor} \psi(t)$, where $t=x-\lfloor x\rfloor \in[0,1)$. Then $f(x+2)=u_{\lfloor x+2\rfloor} \varphi(t)+v_{\lfloor x+2\rfloor} \psi(t)$. Since $\lfloor x+2\rfloor=$ $\lfloor x\rfloor+2$ and thus $x+2-\lfloor x+2\rfloor=x-\lfloor x\rfloor$, we obtain $f(x+2)=\left(u_{\lfloor x+1\rfloor}+u_{\lfloor x\rfloor}\right) \varphi(t)+\left(v_{\lfloor x+1\rfloor}+\right.$ $\left.v_{\lfloor x\rfloor}\right) \psi(t)=f(x+1)+f(x)$, i.e., $f(x)$ is a Fibonacci function.

Note that Example 2.2 is a special case of Example 2.3 with $\varphi(t)=1, \psi(t)=t$. By choosing of the suitable functions $\varphi$ and $\psi$, e.g., $\varphi(t)=\frac{1}{2}, \psi(t)=\frac{1}{2} t^{2}$, we obtain another example.

Remark Note that the Fibonacci function $f(x)$ in Example 2.3 does not have the form $f(x)=\lambda a^{x}$, which is monotone in any case. In fact, let $\left\{u_{n}\right\}_{n=-\infty}^{\infty}$ and $\left\{v_{n}\right\}_{n=-\infty}^{\infty}$ be full Fibonacci sequences with $u_{0}=u_{1}=1, v_{0}=\frac{1}{2}, v_{1}=2$. Then $u_{3}=3, u_{-5}=-3$, and $v_{3}=4.5$, $v_{-5}=6$. Thus $f(-4.8)=u_{\lfloor-4.8\rfloor}+v_{\lfloor-4.8\rfloor}(-4.8-\lfloor-4.8\rfloor)=u_{-5}+v_{-5}(0.2)=-1.8<0$ and $f(3.2)=u_{\lfloor 3.2\rfloor}+v_{\lfloor 3.2\rfloor}(3.2-\lfloor 3.2\rfloor)=u_{3}+v_{3}(0.2)=3.9>0$. Assume $f(x)=\lambda a^{x}$ where $a>0$. If $\lambda>0$, then $f(x)>0$ and $f(-4.8)=-1.8<0$. If $\lambda<0$, then $f(x)<0$ and $f(3.2)=3.9>0$. If $\lambda=0$, then $f(x)=0$. Thus $f(x)$ does not have the form $f(x)=\lambda a^{x}$, which is monotone in any case.

Using the notions of an $f$-even function and an $f$-odd function, we obtain many Fibonacci functions as discussed in [7].

Definition 2.4 ([7]) Let $a(x)$ be a real-valued function of a real variable such that if $a(x) h(x) \equiv 0$ and $h(x)$ is continuous, then $h(x) \equiv 0$. The map $a(x)$ is said to be an $f$-even function (resp., $f$-odd function) if $a(x+1)=a(x)$ (resp., $a(x+1)=-a(x)$ ) for any $x \in \mathbf{R}$. 
Theorem 2.5 ([7]) Let $f(x)=a(x) g(x)$ be a function, where $a(x)$ is an $f$-even function and $g(x)$ is a continuous function. Then $f(x)$ is a Fibonacci function if and only if $g(x)$ is a Fibonacci function.

Example 2.6 ([7]) It follows from Example 2.1 that $g(x)=\left(\frac{1+\sqrt{5}}{2}\right)^{x}$ is a Fibonacci function. Since $a(x)=x-\lfloor x\rfloor$ is an $f$-even function, by Theorem 2.5, $f(x)=a(x) g(x)=(x-\lfloor x\rfloor)\left(\frac{1+\sqrt{5}}{2}\right)^{x}$ is a Fibonacci function.

Example 2.7 ([7]) If we define $a(x)=1$ if $x$ is rational and $a(x)=-1$ if $x$ is irrational, then $a(x+1)=a(x)$ for any $x \in \mathbf{R}$. Also, if $a(x) h(x) \equiv 0$, then $h(x) \equiv 0$ whether or not $h(x)$ is continuous. Thus $a(x)$ is an $f$-even function. In Example 2.6, we have seen that $f(x)=$ $(x-\lfloor x\rfloor)\left(\frac{1+\sqrt{5}}{2}\right)^{x}$ is a Fibonacci function. By applying Theorem 2.5 , the map defined by

$$
a(x) f(x)= \begin{cases}(x-\lfloor x\rfloor)\left(\frac{1+\sqrt{5}}{2}\right)^{x} & \text { if } x \in Q, \\ -(x-\lfloor x\rfloor)\left(\frac{1+\sqrt{5}}{2}\right)^{x} & \text { otherwise }\end{cases}
$$

is also a Fibonacci function.

Corollary 2.8 ([7]) Let $f(x)=a(x) g(x)$ be a function, where $a(x)$ is an $f$-odd function and $g(x)$ is a continuous function. Then $f(x)$ is a Fibonacci function if and only if $g(x)$ is an odd Fibonacci function.

Example 2.9 ([7]) The function $g(x)=\left(\frac{\sqrt{5}-1}{2}\right)^{x}$ is an odd Fibonacci function. Since $a(x)=$ $\sin (\pi x)$ is an $f$-odd function, by Corollary 2.8 , we can see that the function $f(x)=$ $\sin (\pi x)\left(\frac{\sqrt{5}-1}{2}\right)^{x}$ is a Fibonacci function.

Note that if a Fibonacci function is differentiable on $\mathbf{R}$, then its derivative is also a Fibonacci function.

Proposition 2.10 ([7]) Let $f$ be a Fibonacci function. If we define $g(x):=f(x+t)$ where $t \in \mathbf{R}$ for any $x \in \mathbf{R}$, then $g$ is also a Fibonacci function.

For example, since $f(x)=\left(\frac{1+\sqrt{5}}{2}\right)^{x}$ is a Fibonacci function, $g(x)=\left(\frac{1+\sqrt{5}}{2}\right)^{x+t}=\left(\frac{1+\sqrt{5}}{2}\right)^{t} f(x)$ is also a Fibonacci function where $t \in \mathbf{R}$.

Theorem 2.11 ([7]) If $f(x)$ is a Fibonacci function, then the limit of the quotient $\frac{f(x+1)}{f(x)}$ exists.

Corollary 2.12 ([7]) If $f(x)$ is a Fibonacci function, then

$$
\lim _{x \rightarrow \infty} \frac{f(x+1)}{f(x)}=\frac{1+\sqrt{5}}{2} .
$$

\section{Fibonacci functions with periodicity}

In this section, we obtain several results on Fibonacci functions using the periodicity.

Proposition 3.1 Let $g(x)$ and $f(x)$ be Fibonaccifunctions with $g(x)=a(x) f(x)$ for some $a(x)$.

Then

$$
\lim _{x \rightarrow \infty} \frac{a(x+1)}{a(x)}=1 .
$$


Proof If $g(x)$ and $f(x)$ are Fibonacci functions, then by Corollary 2.12 we obtain

$$
\begin{aligned}
\frac{1+\sqrt{5}}{2} & =\lim _{x \rightarrow \infty} \frac{g(x+1)}{g(x)} \\
& =\lim _{x \rightarrow \infty} \frac{a(x+1) f(x+1)}{a(x) f(x)} \\
& =\lim _{x \rightarrow \infty} \frac{a(x+1)}{a(x)} \lim _{x \rightarrow \infty} \frac{f(x+1)}{f(x)} \\
& =\frac{1+\sqrt{5}}{2} \lim _{x \rightarrow \infty} \frac{a(x+1)}{a(x)} .
\end{aligned}
$$

This proves the proposition.

Corollary 3.2 Let $g(x)$ and $f(x)$ be Fibonacci functions with $g(x)=a(x) f(x)$ for some $a(x)$.

Then

$$
\lim _{x \rightarrow \infty} \frac{a(x+k)}{a(x)}=1
$$

for all natural numbers $k$.

Proof It follows from the following equation:

$$
\begin{aligned}
\lim _{x \rightarrow \infty} \frac{a(x+k)}{a(x)} & =\lim _{x \rightarrow \infty} \frac{a(x+k) a(x+k-1) \cdots a(x+1)}{a(x+k-1) a(x+k-2) \cdots a(x)} \\
& =\lim _{x \rightarrow \infty} \frac{a(x+1) f(x+1)}{a(x) f(x)} \\
& =\lim _{x \rightarrow \infty} \frac{a(x+k)}{a(x+k-1)} \lim _{x \rightarrow \infty} \frac{a(x+k-1)}{a(x+k-2)} \cdots \lim _{x \rightarrow \infty} \frac{a(x+1)}{a(x)} \\
& =1 .
\end{aligned}
$$

Corollary 3.3 Let $g(x)$ and $f(x)$ be Fibonacci functions with $g(x)=a(x) f(x)$ for some $a(x)$. If $y>0$, then

$$
\lim _{x \rightarrow \infty} \frac{a(x+y)}{a(x)}=\lim _{x \rightarrow \infty} \frac{a(x+y)}{a(x+y-\lfloor y\rfloor)} .
$$

Proof It follows from Corollary 3.2 that

$$
\begin{aligned}
\lim _{x \rightarrow \infty} \frac{a(x+y)}{a(x)} & =\lim _{x \rightarrow \infty} \frac{a(x+y) a(x+y-\lfloor y\rfloor)}{a(x+y-\lfloor y\rfloor) a(x)} \\
& =\lim _{x \rightarrow \infty} \frac{a(x+y)}{a(x+y-\lfloor y\rfloor)} \lim _{x \rightarrow \infty} \frac{a(x+y-\lfloor y\rfloor)}{a(x)} \\
& =\lim _{x \rightarrow \infty} \frac{a(x+y-\lfloor y\rfloor+\lfloor y\rfloor)}{a(x+y-\lfloor y\rfloor)} \lim _{x \rightarrow \infty} \frac{a(x+y-\lfloor y\rfloor)}{a(x)} \\
& =\lim _{x \rightarrow \infty} \frac{a(x+y)}{a(x+y-\lfloor y\rfloor)} .
\end{aligned}
$$


A map $t(x)$ is said to be ultimately periodic of period $p>0$ if

$$
\lim _{x \rightarrow \infty} \frac{t(x+p)}{t(x)}=1 .
$$

Note that $a(x)$ discussed in Proposition 3.1 is ultimately periodic of period 1 .

Example 3.4 Let $t(x):=m x+b$. If $m \neq 0$, then $\lim _{x \rightarrow \infty} \frac{t(x+p)}{t(x)}=\lim _{x \rightarrow \infty} \frac{m(x+p)+b}{m x+b}=1$, showing that $t(x)$ is ultimately periodic of period $p$ for all $p>0$.

Using Example 3.4, we obtain the following example.

Example 3.5 If $t(x):=a_{n} x^{n}+a_{n-1} x^{n-1}+\cdots+a_{0}$, then $t(x)$ is ultimately periodic of period $p$ for all $p>0$.

Example 3.6 If $t(x):=\sin x$, then $\frac{\sin (x+p)}{\sin x}=\frac{\sin x \cos p+\cos x \sin p}{\sin x}=\cos p+\sin p \cot x$. It follows that

$$
\lim _{x \rightarrow \infty} \frac{\sin (x+p)}{\sin x}=\cos p+\sin p \lim _{x \rightarrow \infty} \cot x .
$$

Since $\lim _{x \rightarrow \infty} \cot x$ does not exist, $t(x)$ is not ultimately periodic of period $p>0$ unless $\sin p=0$ and $\cos p=1$, i.e., $p=2 k \pi$ for any integer $k>0$.

Proposition 3.7 If $a(x)$ and $b(x)$ are ultimately periodic of period $p>0$, then $\alpha a(x)+\beta b(x)$ is also ultimately periodic of period $p>0$ for all $\alpha, \beta>0$.

Proof Since $a(x)$ and $b(x)$ are ultimately periodic of period $p>0$, there exist $\epsilon_{1}(x), \epsilon_{2}(x)>0$ such that $\frac{a(x+p)}{a(x)}=1+\epsilon_{1}(x)$ and $\frac{b(x+p)}{b(x)}=1+\epsilon_{2}(x)$ where $\epsilon_{i}(x) \rightarrow 0(i=1,2)$. We know that $\frac{1+\epsilon_{1}(x)}{1+\epsilon_{2}(x)}=1+\epsilon(x)$ for some $\epsilon(x)$. In fact, $\epsilon(x)=\frac{\epsilon_{2}(x)-\epsilon_{1}(x)}{1+\epsilon_{1}(x)} \rightarrow 0$. This shows that

$$
\begin{aligned}
\frac{\alpha a(x+p)+\beta b(x+p)}{\alpha a(x)+\beta b(x)} & =\frac{1+\frac{\beta b(x+p)}{\alpha a(x+p)}}{1+\frac{\beta b(x)}{\alpha a(x)}} \frac{\alpha a(x+p)}{\alpha a(x)} \\
& =\frac{1+\frac{\beta}{\alpha} \frac{\left(1+\epsilon_{2}(x)\right) b(x)}{\left.1+\epsilon_{1}(x)\right) a(x)}}{1+\frac{\beta}{\alpha} \frac{b(x)}{a(x)}} \frac{a(x+p)}{a(x)} \\
& =\frac{1+\frac{\beta}{\alpha}(1+\epsilon(x)) \frac{b(x)}{a(x)}}{1+\frac{\beta}{\alpha} \frac{b(x)}{a(x)}} \frac{a(x+p)}{a(x)} \\
& \rightarrow \frac{a(x+p)}{a(x)} \\
& \rightarrow 1 .
\end{aligned}
$$

This proves the proposition.

Proposition 3.8 If $a(x)$ and $b(x)$ are ultimately periodic of period $p>0$, then $a(x) b(x)$ is also ultimately periodic of period $p>0$.

Proof It follows from the following equation:

$$
\lim _{x \rightarrow \infty} \frac{a(x+p) b(x+p)}{a(x) b(x)}=\lim _{x \rightarrow \infty} \frac{a(x+p)}{a(x)} \lim _{x \rightarrow \infty} \frac{b(x+p)}{b(x)}=1 .
$$


Theorem 3.9 The collection $U_{p}$ of all functions which are ultimately periodic of period $p>0$ forms an algebra.

Proof It follows immediately from Propositions 3.7 and 3.8.

Proposition 3.10 If $a(x) \in U_{p}$ and $a(x) \neq 0$ for all $x \in[\lambda, \infty)$ for some $\lambda$, then $\frac{1}{a(x)} \in U_{p}$.

Proof It follows from the following equation:

$$
\lim _{x \rightarrow \infty} \frac{\frac{1}{a(x+p)}}{\frac{1}{a(x)}}=\lim _{x \rightarrow \infty} \frac{a(x)}{a(x+p)}=1 .
$$

Proposition 3.11 If $a(x) \in U_{p}$, then $\frac{1}{a(x)} \in U_{k p}$ for all natural numbers $k$.

Proof The proof is similar to that of Corollary 3.2.

A map $f$ defined on the set of all real numbers $\mathbf{R}$ is said to be periodic of period $p>0$ if $f(x+p)=f(x)$ for all $x \in \mathbf{R}$. It is obvious that every map of period of periodic 1 is ultimately periodic of period 1 .

Proposition 3.12 Let $f(x)$ be a Fibonacci function and let $a(x)$ be periodic of period 1 . If $g(x):=a(x) f(x)$, then $g(x)$ is a Fibonacci function.

Proof Given $x \in \mathbf{R}$, since $a(x)$ is periodic of period 1, we have

$$
\begin{aligned}
g(x+2) & =a(x+2) f(x+2) \\
& =a(x+2) f(x+1)+a(x) f(x) \\
& =a(x+1) f(x+1)+a(x) f(x) \\
& =g(x+1)+g(x) .
\end{aligned}
$$

We ask the following question: Are there a Fibonacci function $f(x)$ and a function $a(x)$ which is ultimately periodic of period 1 but not periodic of period 1 such that $g(x)=$ $a(x) f(x)$ is also a Fibonacci function?

Theorem 3.13 Let $f(x), g(x)$ be Fibonacci functions with $g(x)=a(x) f(x)$. If $a(x+1) \neq a(x)$ for all $x \in \mathbf{R}$, then

$$
\lim _{x \rightarrow \infty} \frac{a(x+2)-a(x)}{a(x+2)-a(x+1)}=-\frac{1+\sqrt{5}}{2} .
$$

Proof Since $a(x+2) \neq a(x)$ for all $x \in \mathbf{R}$, we have

$$
\begin{aligned}
a(x+2)[f(x+1)+f(x)] & =a(x+2) f(x+2) \\
& =g(x+2) \\
& =a(x+1) f(x+1)+a(x) f(x) .
\end{aligned}
$$


It follows that $[a(x+2)-a(x+1)] f(x+1)=-[a(x+2)-a(x)] f(x)$, which implies

$$
\frac{f(x+1)}{f(x)}=-\frac{a(x+2)-a(x)}{a(x+2)-a(x+1)} .
$$

By Corollary 2.12, we obtain

$$
\lim _{x \rightarrow \infty} \frac{a(x+2)-a(x)}{a(x+2)-a(x+1)}=-\lim _{x \rightarrow \infty} \frac{f(x+1)}{f(x)}=-\frac{1+\sqrt{5}}{2}
$$

proving the theorem.

If we let $a(x):=x^{2}$, then $\lim _{x \rightarrow \infty} \frac{(x+2)^{2}-x^{2}}{(x+2)^{2}-(x+2)^{2}}=\lim _{x \rightarrow \infty} \frac{4 x+4}{2 x+3}=2 \neq-\frac{1+\sqrt{5}}{2}$. This shows that $g(x)=x^{2} f(x)$ cannot be a Fibonacci function for any Fibonacci function $f(x)$.

Note that $a(x)$ cannot be an increasing function on $[\lambda, \infty)$ for some $\lambda \in \mathbf{R}$. In fact, we suppose that there is an $\lambda \in \mathbf{R}$ such that $a(x)<a(x+1)$ for all $x \geq \lambda$. Then $a(x+2)-a(x+1)<$ $a(x+2)-a(x)$. It follows that $1 \leq \lim _{x \rightarrow \infty} \frac{a(x+2)-a(x)}{a(x+2)-a(x+1)}=-\frac{1+\sqrt{5}}{2}$, a contradiction.

Given $\lambda \in \mathbf{R}$, if we let $\Phi:=\frac{1+\sqrt{5}}{2}$ and we let

$$
\frac{a(x+2)-a(x)}{a(x+2)-a(x+1)}=-\Phi
$$

then $a(x+2)-a(x)=\Phi a(x+1)-\Phi a(x+2)$. It follows that

$$
\begin{aligned}
a(x+2) & =\frac{\Phi}{1+\Phi} a(x+1)+\frac{1}{1+\Phi} a(x) \\
& =\frac{1}{\Phi} a(x+1)+\frac{1}{\Phi^{2}} a(x) .
\end{aligned}
$$

Theorem 3.14 Let $f(x)$ be a Fibonacci function and let $a(x)$ be a map with condition (2). If $g(x):=a(x) f(x)$ is a Fibonacci function for all $x \geq \lambda$, then

$$
f(x+1)=\Phi f(x)
$$

for all $x \geq \lambda$.

Proof Let $a(x)$ be a function satisfying the condition (2). Since $f(x)$ is a Fibonacci function, we have the following:

$$
\begin{aligned}
g(x+2)= & a(x+2) f(x+2) \\
= & {\left[\frac{\Phi}{1+\Phi} a(x+1)+\frac{1}{1+\Phi} a(x)\right][f(x+1)+f(x)] } \\
= & \frac{\Phi}{1+\Phi} a(x+1) f(x+1)+\frac{\Phi}{1+\Phi} a(x+1) f(x) \\
& +\frac{1}{1+\Phi} a(x) f(x+1)+\frac{1}{1+\Phi} a(x) f(x) \\
= & g(x+1)+g(x)-\frac{1}{1+\Phi} g(x+1)+\frac{\Phi}{1+\Phi} a(x+1) f(x) \\
& +\frac{1}{1+\Phi} a(x) f(x+1)-\frac{\Phi}{1+\Phi} g(x) .
\end{aligned}
$$


Since $g(x)$ is also a Fibonacci function, we obtain

$$
\frac{\Phi}{1+\Phi} a(x+1) f(x)+\frac{1}{1+\Phi} a(x) f(x+1)=\frac{1}{1+\Phi} g(x+1)+\frac{\Phi}{1+\Phi} g(x) .
$$

It follows that

$$
\frac{1}{1+\Phi}[a(x) f(x+1)-g(x+1)]=\frac{\Phi}{1+\Phi}[g(x)-a(x+1) f(x)]
$$

This shows that

$$
\begin{aligned}
\Phi & =\frac{a(x) f(x+1)-g(x+1)}{g(x)-a(x+1) f(x)} \\
& =\frac{a(x) f(x+1)-a(x+1) f(x+1)}{a(x) f(x)-a(x+1) f(x)} \\
& =\frac{f(x+1)}{f(x)}
\end{aligned}
$$

for all $x \geq \lambda$.

\section{Exponential Fibonacci functions}

Consider a map $T(x):=\frac{\ln (1+x)}{\ln x}$ with domain $D=(0,1) \cup(1, \infty)$. If we let $D^{*}:=(-\infty, 0) \cup$ $(1, \infty)$, then $T: D \rightarrow D^{*}$ is a bijective function. If $x \in \mathbf{R}$ such that $x^{2}=x+1$, then $T^{-1}(2)=$ $x=\Phi$.

Theorem 4.1 Let $f: \mathbf{R} \rightarrow \mathbf{R}$ be a map such that $f(x+2)=2 f(x+1)-f(x)$ and let $A:=$ $\left(T^{-2}(2)\right)^{\frac{1}{\beta}}$ where $\beta \neq 0$. Then $g(x):=A^{f(x)}$ is a Fibonacci function.

Proof If we assume $g(x)$ is a Fibonacci function, then

$$
A^{f(x+2)}=A^{f(x+1)}+A^{f(x)}
$$

where $A>0$. Then $A^{f(x+2)-f(x)}=A^{f(x+1)-f(x)}+1$. Let $\alpha(x):=f(x+2)-f(x)$ and let $\beta(x):=$ $f(x+1)-f(x)$. Then $A^{\alpha(x)}=A^{\beta(x)}+1$. It follows that

$$
\begin{aligned}
\ln \left(A^{\beta(x)}+1\right) & =\ln \left(A^{\alpha(x)}\right) \\
& =\alpha(x) \ln A \\
& =\frac{\alpha(x)}{\beta(x)} \ln \left(A^{\beta(x)}\right) .
\end{aligned}
$$

This shows that

$$
\frac{\alpha(x)}{\beta(x)}=\frac{\ln \left(A^{\beta(x)}+1\right)}{\ln \left(A^{\beta(x)}\right)}=T\left(A^{\beta(x)}\right) .
$$

Hence $\frac{\alpha(x)}{\beta(x)} \in D^{*}$ and $A^{\beta(x)}=T^{-1}\left(\frac{\alpha(x)}{\beta(x)}\right)$, i.e., $A=\left[T^{-1}\left(\frac{\alpha(x)}{\beta(x)}\right)\right]^{\frac{1}{\beta(x)}} . A$ is a constant, $\frac{\alpha(x)}{\beta(x)}$ and $\frac{1}{\beta(x)}$ are also constants for all $x \in \mathbf{R}$. Hence there exists $K \in D^{*}$ such that $K=\frac{\alpha(x)}{\beta(x)}=\frac{f(x+2)-f(x)}{f(x+1)-f(x)}$. It 
follows that

$$
f(x+2)=K f(x+1)+(1-K) f(x) .
$$

If we let $\beta(x):=\beta$, a constant, for all $x \in \mathbf{R}$, then $\alpha(x)=K \beta(x)=K \beta$. It follows that $(K-$ 1) $\beta=\alpha-\beta=[f(x+2)-f(x)]-[f(x+1)-f(x)]=f(x+2)-f(x+1)=\beta(x+1)=\beta$, i.e., $K=2$. This shows that $g(x)=\left[\left(T^{-2}(2)\right)^{\frac{1}{\beta}}\right]^{f(x)}=\left[\Phi^{\frac{1}{\beta}}\right]^{f(x)}$ is a Fibonacci function where $\beta \neq 0$.

The map $T$ discussed above is useful for the following proposition.

Proposition 4.2 If $g(x)=A(x)^{f(x)}$ is a Fibonacci function where $A(x)>0$, then there exists $\gamma(x) \in D^{*}$ such that

$$
\frac{g(x+2)}{g(x)}=\left[\frac{g(x+1)}{g(x)}\right]^{\gamma(x)} .
$$

Proof If $g(x)=A(x)^{f(x)}, A(x)>0$, then $g(x)>0$. Assume

$$
\frac{g(x+2)}{g(x)}=\left[\frac{g(x+1)}{g(x)}\right]^{\gamma(x)}
$$

for some $\gamma(x)$. If we let $B(x):=\frac{g(x+1)}{g(x)}$, then

$$
\begin{aligned}
B(x)^{\gamma(x)} & =\frac{g(x+2)}{g(x)} \\
& =\frac{g(x+1)+g(x)}{g(x)} \\
& =B(x)+1 .
\end{aligned}
$$

It follows that

$$
\begin{aligned}
\gamma(x) & =\frac{\ln (B(x)+1)}{\ln B(x)} \\
& =\frac{\ln \left(\frac{g(x+1)}{g(x)}+1\right)}{\ln \left(\frac{g(x+1)}{g(x)}\right)} \\
& =T(B(x)) \in D^{*},
\end{aligned}
$$

proving the proposition.

The converse of Proposition 4.2 need not be true in general. If $g(x)=e^{x}$, then it is not a Fibonacci function. If we let $B(x):=\frac{g(x+1)}{g(x)}$, then $B(x)=e$ and $\gamma(x)=\frac{\ln (B(x)+1)}{\ln B(x)}=\ln (e+1)$. It follows that $B(x)^{\gamma(x)}=B(x)+1$.

We construct Fibonacci functions directly as follows.

Theorem 4.3 Let $\beta \neq 0$ be a constant and let $f$ be a map defined on $[0, \infty)$. Define a map $f_{\beta}: \mathbf{R} \rightarrow \mathbf{R}$ by $f_{\beta}(x):=\lfloor x\rfloor \beta+f(x-\lfloor x\rfloor)$ for all $x \in \mathbf{R}$. Then $g(x):=\left[\Phi^{1 / \beta}\right]^{f_{\beta}(x)}$ is a Fibonacci function. 
Proof If we let $A:=\Phi^{1 / \beta}$, then $g(x)=A^{f_{\beta}(x)}=A^{\lfloor x\rfloor \beta+f(x-\lfloor x\rfloor)}=\Phi^{\lfloor x\rfloor} A^{f(x-\lfloor x\rfloor)}$. It follows that

$$
\begin{aligned}
g(x+2) & =A^{\lfloor x+2\rfloor \beta+f(x+2-\lfloor x+2\rfloor)} \\
& =\Phi^{2} \Phi^{\lfloor x\rfloor} A^{f(x-\lfloor x\rfloor)} \\
& =[\Phi+1] g(x) \\
& =\Phi g(x)+g(x) \\
& =g(x+1)+g(x),
\end{aligned}
$$

proving the theorem.

Proposition 4.4 There is no Fibonacci function $f(x)$ such that $g(x)=A^{f(x)}, A>0$ where $f(x)$ is differentiable and $g(x)$ is a Fibonacci function.

Proof Assume that $f(x)$ is a Fibonacci function. Since $f(x)$ is differentiable, we have

$$
f^{\prime}(x+2)=f^{\prime}(x+1)+f^{\prime}(x) .
$$

Since $g(x)$ is a Fibonacci function, we have $g(x+2)=g(x+1)+g(x)$. Since $g(x)=A^{f(x)}$ and $f(x)$ is differentiable, $g^{\prime}(x+2)=g^{\prime}(x+1)+g^{\prime}(x)$, i.e., $g^{\prime}(x)$ is also a Fibonacci function. It follows from $g^{\prime}(x)=g(x) \ln A f^{\prime}(x)$ that

$$
\begin{aligned}
g(x+2) \ln A f^{\prime}(x+2) & =g^{\prime}(x+2) \\
& =g^{\prime}(x+1)+g^{\prime}(x) \\
& =g(x+1) \ln A f^{\prime}(x+1)+g(x) \ln A f^{\prime}(x) .
\end{aligned}
$$

Since $g(x+2) \neq 0$, we obtain

$$
f^{\prime}(x+2)=\frac{g(x+1)}{g(x+2)} f^{\prime}(x+1)+\frac{g(x)}{g(x+2)} f^{\prime}(x) .
$$

By (4) and (5), we obtain

$$
\left[\frac{g(x+1)}{g(x+2)}-1\right] f^{\prime}(x+1)+\left[\frac{g(x)}{g(x+1)}-1\right] f^{\prime}(x)=0 \text {. }
$$

This shows that

$$
\frac{f^{\prime}(x+1)}{f^{\prime}(x)}=\frac{g(x)-g(x+2)}{g(x+2)-g(x+1)}=-\frac{g(x+1)}{g(x)} .
$$

It follows that

$$
\Phi=\lim _{x \rightarrow \infty} \frac{f^{\prime}(x+1)}{f^{\prime}(x)}=\lim _{x \rightarrow \infty}-\frac{g(x+1)}{g(x)}=-\Phi,
$$

a contradiction. Hence $f(x)$ should not be a Fibonacci function. 
Competing interests

The authors declare that they have no competing interests.

\section{Authors' contributions}

All authors conceived of the study, participated in its design and coordination, drafted the manuscript, participated in the sequence alignment, and read and approved the final manuscript.

\section{Author details}

${ }^{1}$ Department of Mathematics, Research Institute for Natural Sciences, Hanyang University, Seoul, 133-791, Korea.

${ }^{2}$ Department of Mathematics, University of Alabama, Tuscaloosa, AL 35487-0350, USA. ${ }^{3}$ Department of Mathematics,

Hallym University, Chuncheon, 200-702, Korea.

\section{Acknowledgements}

The authors are grateful for a referee's valuable suggestions and help. This research was supported by Hallym University Research Fund 2014 (HRF-201401-008).

Received: 11 June 2014 Accepted: 6 November 2014 Published: 25 Nov 2014

References

1. Atanassov, KT, Atanassova, V, Shannon, AG, Turner, JC: New Visual Perspectives on Fibonacci Numbers. World Scientific, River Edge (2002)

2. Dunlap, RA: The Golden Ratio and Fibonacci Numbers. World Scientific, River Edge (1997)

3. Kim, HS, Neggers, J: Fibonacci means and golden section mean. Comput. Math. Appl. 56, 228-232 (2008)

4. Jung, SM: Hyers-Ulam stability of Fibonacci functional equation. Bull. Iran. Math. Soc. 35, 217-227 (2009)

5. Han, JS, Kim, HS, Neggers, J: Fibonacci sequences in groupoids. Adv. Differ. Equ. 2012, 19 (2012). doi:10.1186/1687-1847-2012-19

6. Kim, HS, Neggers, J, So, KS: Generalized Fibonacci sequences in groupoids. Adv. Differ. Equ. 2013, 26 (2013). doi:10.1186/1687-1847-2013-26

7. Han, JS, Kim, HS, Neggers, J: On Fibonacci functions with Fibonacci numbers. Adv. Differ. Equ. 2012, 126 (2012). doi:10.1186/1687-1847-2012-126

10.1186/1687-1847-2014-293

Cite this article as: Kim et al.: On Fibonacci functions with periodicity. Advances in Difference Equations 2014, $2014: 293$

\section{Submit your manuscript to a SpringerOpen ${ }^{\circ}$ journal and benefit from:}

- Convenient online submission

- Rigorous peer review

Immediate publication on acceptance

- Open access: articles freely available online

- High visibility within the field

- Retaining the copyright to your article 